\title{
Universal properties of highly frustrated quantum magnets in strong magnetic fields
}

\author{
Oleg Derzhko ${ }^{1,2,3}$, Johannes Richter ${ }^{2,3}$, Andreas Honecker ${ }^{4}$, \\ and Heinz-Jürgen Schmidt ${ }^{5}$ \\ ${ }^{1}$ Institute for Condensed Matter Physics, National Academy of Sciences of Ukraine \\ 1 Svientsitskii Str., L'viv 79011, Ukraine \\ ${ }^{2}$ Max-Planck-Institut für Physik komplexer Systeme, 38 Nöthnitzer Str., Dresden 01187, Germany \\ ${ }^{3}$ Institut für Theoretische Physik, Universität Magdeburg, P.O. Box 4120, Magdeburg 39016, Germany \\ E-mail: Johannes.Richter@Physik.Uni-Magdeburg.DE \\ ${ }^{4}$ Institut für Theoretische Physik, Georg-August-Universität Göttingen \\ 1 Friedrich-Hund-Platz, Göttingen 37077, Germany \\ ${ }^{5}$ Fachbereich Physik, Universität Osnabrück, 7 Barbara Str., Osnabrück 49069, Germany
}

Received December 12, 2006

\begin{abstract}
The purpose of the present paper is two-fold. On the one hand, we review some recent studies on the low-temperature strong-field thermodynamic properties of frustrated quantum spin antiferromagnets which admit the so-called localized-magnon eigenstates. One the other hand, we provide some complementary new results. We focus on the linear independence of the localized-magnon states, the estimation of their degeneracy with the help of auxiliary classical lattice-gas models and the analysis of the contribution of these states to thermodynamics.
\end{abstract}

PACS: 75.10.Jm Quantized spin models;

75.45. $\mathbf{j}$ Macroscopic quantum phenomena in magnetic systems;

75.50.Ee Antiferromagnetics.

Keywords: frustrated Heisenberg antiferromagnets, strong magnetic field, localized-magnon states, classical lattice-gas models.

\section{Introduction}

Antiferromagnetically interacting quantum Heisenberg spins on regular geometrically frustrated lattices have attracted much interest over the past few decades [1-3]. On the one hand, there has been tremendous recent progress in synthesizing corresponding magnetic materials [4]. On the other hand, geometrically frustrated quantum antiferromagnets are an excellent play-ground for studying novel quantum many-body phenomena. We mention quantum spin-liquid phases, order-by-disorder phenomena, lattice instabilities to name just a few. Application of an external magnetic field to a frustrated quantum Heisenberg antiferromagnet introduces a new competition between interactions in the spin system that may lead to further interesting phenomena. As an example we mention the half-magnetization plateau stabilized by structural distortion in the pyrochlore lattice $[5,6]$.

The theoretical investigation of frustration effects in quantum spin antiferromagnets usually meets new difficulties; e.g., the quantum Monte Carlo method suffers from the sign problem for frustrated systems. However, it is amazing that just owing to geometrical frustration some possibilities for rigorous analysis emerge. Recently, it has been recognized that many geometrically frustrated lattices (including the kagomé lattice, the checkerboard lattice, and the pyrochlore lattice) admit a simple class of exact eigenstates christened localized magnons [7,8]. These states become the ground states in strong magnetic fields and they are relevant for many physical properties of a wide class of highly frustrated quantum antiferromagnets in the low-temperature strong-field regime [7-23]. In 
particular, the localized-magnon states are responsible for magnetization jumps which the ground-state magnetization curve exhibits at the saturation field $[7,8,10,15-17,23]$, may lead to a high-field spin-Peierls lattice instability $[11,18,19]$, and imply a residual ground-state entropy at the saturation field [12-14]. Moreover, these states dominate the low-temperature thermodynamics in the vicinity of the saturation field $[12-14,20,22,24]$ and may lead to an order-disorder phase transition of purely geometrical origin $[13,22,24]$.

This paper focusses on new developments concerning localized-magnon effects. We mention several reviews on this subject $[3,24,25]$ which, however, do not cover some recent studies on the universal thermodynamic behavior which emerges at low temperatures around the saturation field. We will therefore concentrate on the results obtained during the last two years [20-22]. In passing, we will provide several complementary new results.

Although the effects of the localized magnons have not been observed experimentally so far, recent studies on the spin-1/2 diamond-chain compound azurite $\mathrm{Cu}_{3}\left(\mathrm{CO}_{3}\right)_{2}(\mathrm{OH})_{2}[26,27]$ and the frustrated quasi-two-dimensional spin-1/2 antiferromagnet $\mathrm{Cs}_{2} \mathrm{CuCl}_{4}$ [28] represent closely related physics.

The present paper is organized as follows. In Sec. 2, we recall some basic results concerning the localized-magnon states which are then used throughout the paper. Further, in Sec. 3, we discuss the linear independence of the localized-magnon states with the smallest localization area [21] and their completeness as a basis of the high-field ground states. Next, in Sec. 4, we discuss the universal thermodynamic behavior which the considered frustrated quantum antiferromagnets exhibit at low temperatures in the vicinity of the saturation field $[20,22]$. We distinguish different types of the universal behavior depending on the specific classical lattice-gas model which represents the low-energy degrees of freedom of the spin system. We focus on several representative models, namely, the diamond chain (hard-monomer universality class), the frustrated two-leg ladder and the kagomé-like chain (one-dimensional hard-dimer universality class), and the frustrated bilayer lattice (two-dimensional hard-square universality class). Finally, in Sec. 5, we summarize and briefly comment on some possibilities of experimental observation of the localized-magnon effects.

\section{Localized-magnon states}

In this paper we study the Heisenberg model on several geometrically frustrated lattices. Some of these lattices are shown in Figs. 1, 2. The Heisenberg Hamiltonian of $N$ quantum spins of length $s$ reads
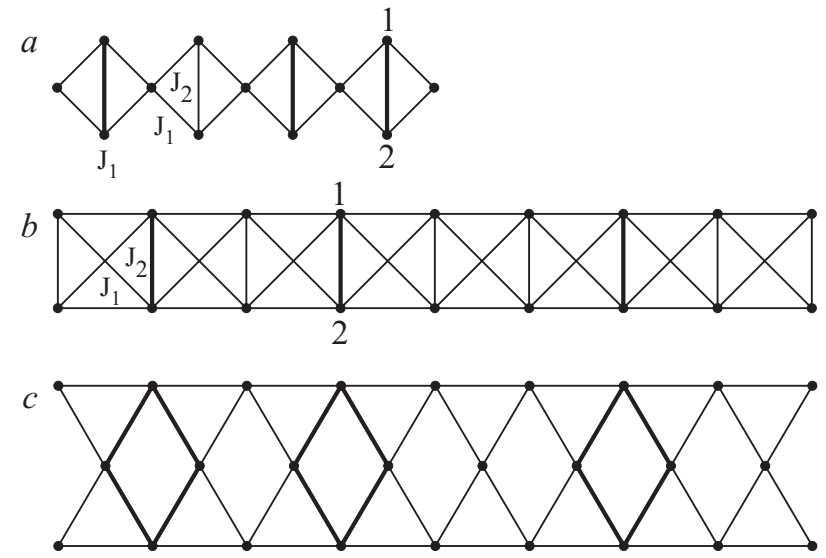

Fig. 1. Three examples of one-dimensional spin lattices admitting localized magnons: the diamond chain (hard-monomer universality class) (a), the frustrated two-leg ladder (one-dimensional hard-dimer universality class) $(b)$, the kagomé-like chain, originally introduced in [29] (one-dimensional hard-dimer universality class) $(c)$.

$$
H=\sum_{(n m)} J_{n m}\left[\frac{1}{2}\left(s_{n}^{+} s_{m}^{-}+s_{n}^{-} s_{m}^{+}\right)+\Delta s_{n}^{z} s_{m}^{z}\right]-h S^{z} .
$$

Here the sum runs over the bonds (edges) which connect the neighboring sites (vertices) on the spin lattice (see Figs. 1,2), $J_{n m}>0$ are the antiferromagnetic exchange integrals between the sites $n$ and $m, \Delta \geq 0$ is the exchange interaction anisotropy parameter, $h$ is the external magnetic field, and $S^{z}=\sum_{n} s_{n}^{z}$ is the $z$-component of the total

spin (magnetization). Note that the operator $S^{z}$ commutes with the Hamiltonian (1). For the examples shown in Figs. 1, $a, b$, and 2,c the exchange integrals take two values, namely, $J_{2}$ for the vertical bonds and $J_{1}$ for all other bonds; for the other lattices shown in Figs. 1, 2 they are uniform $J_{n m}=J$. The exchange integrals $J_{1}$ and $J_{2}$ may also be assumed to satisfy certain relations, see, e.g., Ref. 20 and below. Although the present analysis can be performed for arbitrary values of $s$ and $\Delta$, in what follows we concentrate on the extreme quantum case $s=1 / 2$ and isotropic interactions $\Delta=1$, in particular while performing exact diagonalization for finite systems [30].

From Refs. 3, 7, 8, 25 we know that the Heisenberg antiferromagnet (1) on the lattices shown in Figs. 1, 2, as well as on some other lattices such as the dimer-plaquette chain, the sawtooth chain, another kagomé-like chain $[8,20,39]$ (one-dimensional systems), the square-kagomé lattice, the star lattice (two-dimensional systems), and the pyrochlore lattice (three-dimensional system), support localized-magnon eigenstates. The magnon may be localized on the (vertical) bond as for the lattices shown in Figs. 1,a,b, and 2,c, on the v-part of the sawtooth chain, or 
$a$
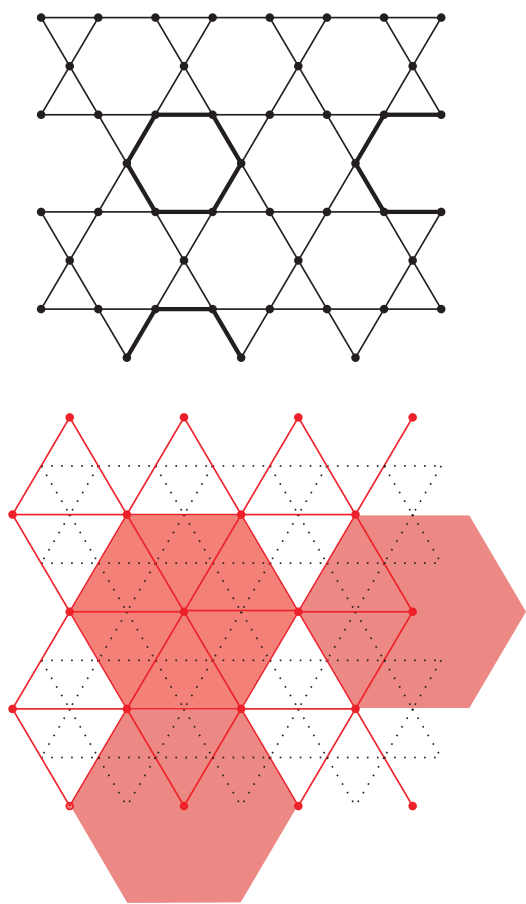

$b$
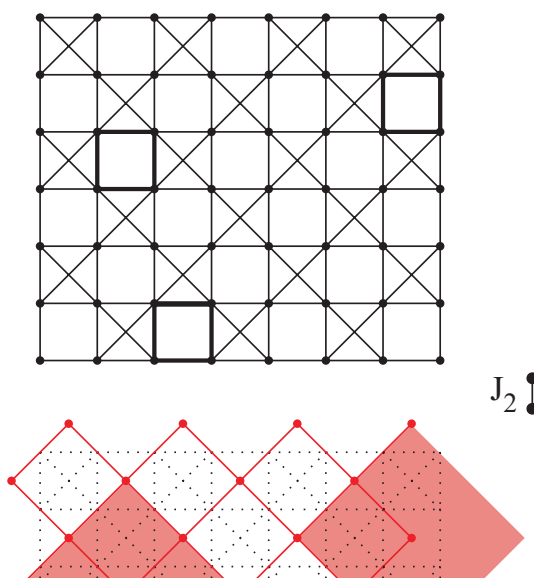

2

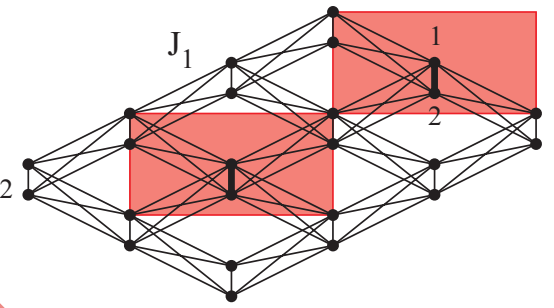

Fig. 2. Three examples of two-dimensional spin lattices admitting localized magnons: the kagomé lattice (hard-hexagon universality class) (a), the checkerboard lattice (large-hard-square universality class) (b), the frustrated bilayer lattice (hard-square universality class) $(c)$. We also show auxiliary lattice-gas models (hard hexagons on a triangular lattice, large hard squares on a square lattice and hard squares on a square lattice) which describe low-energy degrees of freedom of the spin models in strong magnetic fields.

on the even polygon (hexagon, square etc.) from which other lattices are built (kagomé, pyrochlore, checkerboard, square-kagomé etc.), see Figs. 1,c, and 2,a,b. Then the explicit expression for the localized-magnon state reads

$$
|1 \operatorname{lm}\rangle=|\operatorname{lm}\rangle_{l}|s, \ldots, s\rangle_{e},
$$

where $|\operatorname{lm}\rangle_{l}=(1 / \sqrt{2})\left(|s\rangle_{1}|s-1\rangle_{2}-|s-1\rangle_{1}|s\rangle_{2}\right)$ for the lattices in which the magnon is trapped on the vertical bond or $|\operatorname{lm}\rangle_{l} \propto \sum_{m=1}^{L}(-1)^{m} s_{m}^{-}|s\rangle_{1} \ldots|s\rangle_{L}$ for the lattices in which the magnon is trapped on the $L$-site polygon and $|s, \ldots, s\rangle_{e}$ denotes the ferromagnetically polarized environment. By direct calculation one can convince oneself that the state (2) is indeed an eigenstate of the Hamiltonian (1) with the eigenvalue $E_{F M}-\epsilon_{1}$ where $E_{F M}$ is the energy of ferromagnetically polarized lattice and

$$
\begin{gathered}
\epsilon_{1}^{\text {diamond }}=s\left(J_{2}+\Delta\left(2 J_{1}+J_{2}\right)\right), \\
\epsilon_{1}^{\text {ladder }}=s\left(J_{2}+\Delta\left(4 J_{1}+J_{2}\right)\right), \quad \epsilon_{1}^{\text {kagomé }}=2 s(1+2 \Delta) J, \\
\text { and } \epsilon_{1}^{\text {bilayer }}=s\left(J_{2}+\Delta\left(8 J_{1}+J_{2}\right)\right)
\end{gathered}
$$

for the diamond chain, the frustrated two-leg ladder, the kagomé-like chain, and the frustrated bilayer lattice, respectively (here we put $h=0$ in (1)).
Alternatively, one may diagonalize the Hamiltonian (1) in the one-magnon subspace with $S^{z}=N s-1$ to realize that one of the magnon excitation branches is flat (dispersionless). For the diamond chain, the frustrated two-leg ladder, the kagomé-like chain, and the frustrated bilayer lattice the dispersionless one-magnon energy is given by $\Lambda_{k}^{\text {diamond }}=-s\left(J_{2}+\Delta\left(2 J_{1}+J_{2}\right)\right)+h, \Lambda_{k}^{\text {ladder }}=$ $=-s\left(J_{2}+\Delta\left(4 J_{1}+J_{2}\right)\right)+h, \quad \Lambda_{k}^{\text {kagomé }}=-2 s(1+2 \Delta) J+h$, and $\Lambda_{\mathbf{k}}^{\text {bilayer }}=-s\left(J_{2}+\Delta\left(8 J_{1}+J_{2}\right)\right)+h$, respectively (note the correspondence of $\Lambda_{k}$ with $\epsilon_{1}$ ). For some lattices (the sawtooth chain, the kagomé-like chains, the kagomé lattice, the checkerboard lattice etc.) the dispersionless magnon band is the lowest one, for other lattices it may become the lowest one if certain relations on the antiferromagnetic exchange constants are imposed. In particular, for the diamond chain and the frustrated two-leg ladder we have to assume $J_{2} \geq 2 J_{1}$, whereas for the frustrated bilayer lattice we have to assume $J_{2} \geq 4 J_{1}$. If equality in the imposed relations holds, the dispersive higher-energy magnon band touches the dispersionless lowest-energy band at some values of the wave-vector (this also occurs for the kagomé-like chains, the kagomé lattice, and the checkerboard lattice, but not for the sawtooth chain). 
We pass to the subspaces with total

$$
S^{z}=N s-2, \ldots, N s-n_{\max } .
$$

Here $n_{\max }$ is the number of the isolated localized magnons, each occupying the smallest possible area, for the closest packing. $n_{\max }$ depends on the lattice and equals $N / 3, N / 4, N / 6$, and $N / 4$ for the diamond chain, the frustrated two-leg ladder, the kagomé-like chain, and the frustrated bilayer lattice, respectively. Evidently, we can construct eigenstates of the Hamiltonian (1) of the form

$$
\begin{gathered}
|2 \operatorname{lm}\rangle=|\operatorname{lm}\rangle_{l_{1}}|\operatorname{lm}\rangle_{l_{2}}|s, \ldots, s\rangle_{e}, \ldots,\left|n_{\max } \operatorname{lm}\right\rangle= \\
=|\operatorname{lm}\rangle_{l_{1}} \ldots|\operatorname{lm}\rangle_{l_{n_{\max }}}|s, \ldots, s\rangle_{e},
\end{gathered}
$$

which in the zero-field case $h=0$ have the energies $E_{F M}-2 \epsilon_{1}, \ldots, E_{F M}-n_{\max } \epsilon_{1}$, respectively, provided that the trapping cells of the localized magnons are isolated from each other. Obviously, there are many other eigenstates in each of these subspaces. The special importance of the localized-magnon states is due to the fact that they may become ground states (or at least low-lying states) in their respective subspaces. In Refs. 7, 9 it was proven under some quite general assumptions that the localizedmagnon states are indeed lowest-energy states in the corresponding sectors of $S^{z}=N s, \ldots, N s-n_{\max }$. More precisely, if we denote the minimal energy within the subspace with $S^{z}=N s-n$ by $E_{\min }(n)$, the following inequality holds

$$
\begin{aligned}
& E_{\min }(n) \geq(1-n) E_{F M}+n E_{\min }(1)= \\
= & E_{F M}-n\left(E_{F M}-E_{\min }(1)\right)=E_{F M}-n \epsilon_{1}
\end{aligned}
$$

for all $n=0,1, \ldots, 2 N s$ and spin- $s$ Heisenberg systems with sufficiently general coupling schemes [7,9]. The energy of the localized $n$-magnon states is given by the expression on the r.h.s. of this inequality (for $n=1, \ldots, n_{\max }$ ) and hence we conclude that the localized magnons are lowest-energy states in the subspaces with $S^{z}=N s-1, \ldots, N s-n_{\max }$.

We note that the localized-magnon states (2), (3) are highly degenerate states. Obviously, the localized magnons of smallest area can be placed on a lattice in many ways. Moreover, for some lattices there are other states which have the same energy as the localized magnons of smallest area. In the following discussion we will concentrate on the checkerboard lattice and refer to Refs. 13,24 for related remarks on the kagomé lattice. First of all we notice that for many lattices, we can construct localized magnons occupying a larger area in addition to the localized magnon of smallest area, see the example in Fig. 3,a. It is important to note that such eigenstates can be viewed as linear combinations of the simpler eigenstates corresponding to the localized magnons of smallest area. Moreover, in two and higher dimensions there may be additional topological effects. In particular, for some lattices one can construct nested objects (defect states), compare the example in Fig. 3, $b$. Fur-
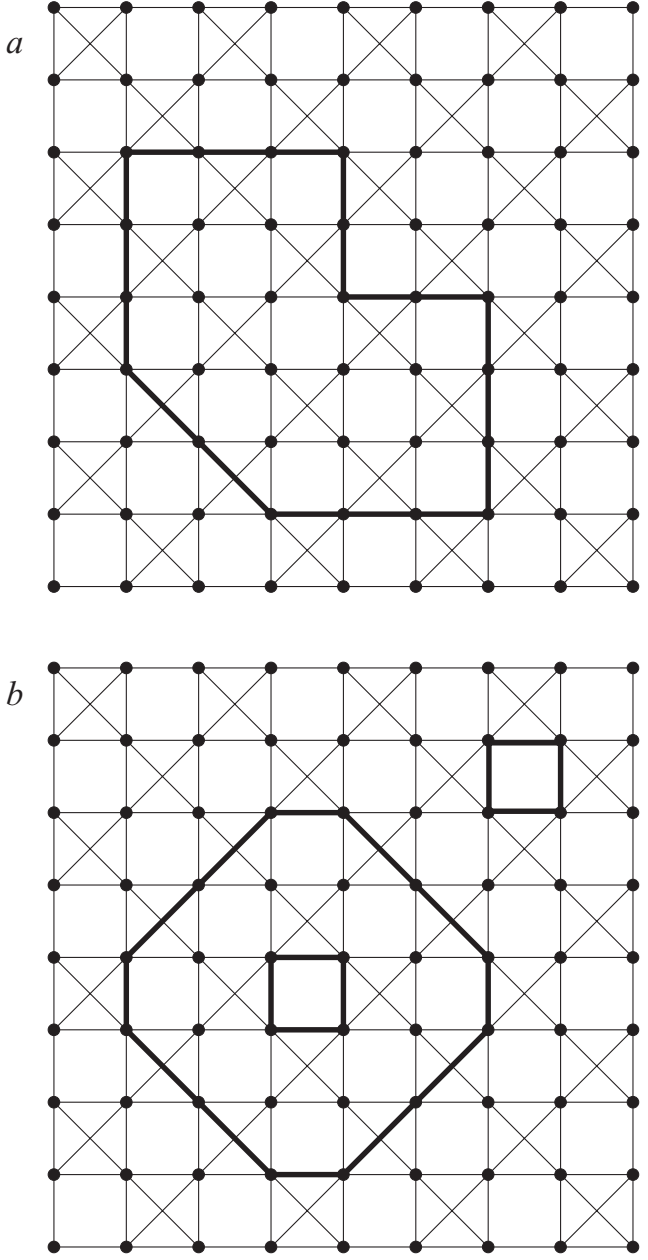

Fig. 3. The checkerboard lattice. A localized magnon which occupies a larger area than the smallest possible one $(a)$. A three-magnon state which is not a large-hard-square state because of the nested «defect» state at the lower left side of the lattice; one magnon is localized on each of the three loops $(b)$.

thermore, for such lattices there may be additional localized magnons with nontrivial winding if periodic boundary conditions are imposed [24]. We shall come back to this issue when discussing the degeneracy of the ground states in the subspaces $S^{z}=N s-2, \ldots, N s-n_{\max }$ for finite systems using exact diagonalization. In particular, we will see that the localized magnons of smallest area linearly span the total ground state space in the sectors $S^{z}=N s-1, \ldots, N s-n_{\max }$ for some lattices (e.g., the diamond chain and the frustrated two-leg ladder with $J_{2}>2 J_{1}$, the sawtooth chain, or the frustrated bilayer lattice with $J_{2}>4 J_{1}$ ).

One is thus led to the task of enumerating all many-localized-magnon states. Let us denote the number of possibilities to put $n$ isolated localized magnons, each occupying the smallest possible area, on a lattice with $N$ sites by $g_{N}(n) \geq 1$. Furthermore note that the energy of a state with $n$ localized magnons reads

$$
E_{n}(h)=E_{F M}-h N s-n\left(\epsilon_{1}-h\right)
$$


in the presence of an external magnetic field. Thus, at the saturation field $h=h_{1}=\epsilon_{1}$ we find that the energy $E_{n}(h)$ does not depend on the number of localized magnons $n$. Hence, the total ground-state degeneracy at $h=h_{1}$ is at least $\mathcal{W}=\sum_{n=0}^{n_{\max }} g_{N}(n)$ provided the considered localized-magnon states are linearly independent (see Ref. 21 and the next section). As a result of this degeneracy one obtains the following universal properties: 1) the ground-state magnetization curve exhibits the jump at $h_{1}$ between the values $N s-n_{\max }$ and $N s$; this jump is accompanied by a plateau at the foot of the jump; 2 ) since $\mathcal{W}$ scales exponentially with $N$ the ground-state entropy per site at $h_{1}$ does not vanish but remains finite, namely at least $k \ln W / N>0$. Moreover, 3) the lattice is unstable with respect to a deformation, which preserves the symmetry required for the existence of localized magnons and lowers the magnetic energy linearly with respect to the displacement. As a result, a field-tuned structural instability may take place. We do not discuss these issues further; more details can be found, e.g., in Refs. 3, 25. Instead we turn to the localized-magnon effects at finite but low temperatures and strong magnetic fields around the saturation field.

\section{Linear independence and completeness}

As discussed in the last section, the localized-magnon states constitute a highly degenerate ground-state manifold at the saturation field $h=h_{1}$. One may expect that these states lead to a dominant contribution to the lowtemperature thermodynamics for magnetic fields in the vicinity of the saturation field. The contribution of the localized-magnon states to the partition function can be written in the form

$$
\begin{aligned}
& Z_{l m}(T, h, N)=\sum_{n=0}^{n_{\max }} g_{N}(n) \exp \left(-\frac{E_{n}(h)}{k T}\right)= \\
= & \exp \left(-\frac{E_{F M}-h N s}{k T}\right) \sum_{n=0}^{n_{\max }} g_{N}(n) \exp \left(\frac{\mu}{k T} n\right),
\end{aligned}
$$

where Eq. (4) was used and $\mu=\epsilon_{1}-h=h_{1}-h$. However, several questions, briefly touched already in the previous section, have to be discussed before determining the degeneracy of the localized-magnon states $g_{N}(n)$ and analyzing the thermodynamic properties on the basis of Eq. (5).

First, we have to clarify whether the set of localized magnons of smallest area is linearly independent in each sector of $S^{z}=N s, \ldots, N s-n_{\max }$. Only if this is the case, all localized magnons of smallest area contribute to the partition function of the spin system and we do not have to take care about the states which are their linear combinations (like the state shown in Fig. 3,a). Bearing in mind the thermodynamic limit $N \rightarrow \infty$ we need at least linear independence in all sectors of $S^{z}$ except probably in a few (finite number) sectors of $S^{z}$.

Although we do not have a general theory of linear independence or dependence of the localized-magnon states of smallest area, a detailed and systematic analysis for a wide class of lattices hosting localized magnons was presented in Ref. 21 including rigorous proofs for lattices in one and two dimensions. We discuss briefly these findings below following Ref. 21. In this discussion we will refer to a localized magnon of smallest area simply by «localized magnon». It appears convenient to group the frustrated lattices into several classes and to examine linear independence for each class separately. Thus, we distinguish the following classes: the orthogonal type (the diamond chain (Fig. 1,a), the dimer-plaquette chain, the frustrated two-leg ladder (Fig. 1,b), the square-kagomé lattice, the frustrated bilayer lattice (Fig. 2,c)); the isolated type (the sawtooth chain, the kagomé-like chain I (Fig. 1,c) and the kagomé-like chain II); the codimension 1 type (the kagomé lattice (Fig. 2,a), the star lattice, the checkerboard lattice (Fig. 2,b)); and the higher codimension type (the pyrochlore lattice).

To examine the linear independence of a finite sequence of $k$ vectors in some Hilbert space it is useful to introduce the $k \times k$ Gram matrix $\mathbf{G}=\left\|G_{i j}\right\|$ of all scalar products. The rank of $\mathbf{G}$ equals the dimension of the linear span of the considered set, i.e., the number of linearly independent states, and the set is linearly independent if $\operatorname{det} \mathbf{G}>0$. We call the dimension of the null space of $\mathbf{G}$ the codimension and hence the codimension will equal the number of independent linear relations between $n$ localized-magnon states.

It can be proven (theorem 1 of Ref. 21) that if the set of localized-magnon states is linearly independent in the sector $S^{z}=N s-1$, then it is also linearly independent in the sectors $S^{z}=N s-n, n=2, \ldots, n_{\max }$. Therefore, in many cases it is sufficient to consider only localized one-magnon states. In particular, for the lattices which belong to the orthogonal type, the cells in which the localized magnons are concentrated are disjoint and any two different localized one-magnon states are orthogonal. As a result, $G_{i j} \propto \delta_{i j}$ and according to the Gram criterion all localized onemagnon states (and hence all localized $n$-magnon states for every $\left.n=1, \ldots, n_{\max }\right)$ are linearly independent. Consider next the lattices which belong to the isolated type. It can be proven (theorem 3 of Ref. 21) that if the trapping cell contains a site which is not contained in any other trapping cell («isolated site») then all localized one-magnon states (and hence all localized $n$-magnon states for every $n=1, \ldots, n_{\max }$ ) are linearly independent.

We pass to the lattices of the codimension 1 type. Suppose that every spin site is contained in exactly two different trapping cells (this is just the case of the lattices of the 
codimension 1 type, e.g., the kagomé lattice (Fig. 2,a), the checkerboard lattice (Fig. 2,b) etc.). Then it can be proven that there is at most one linear relation between localized one-magnon states (theorem 4 of Ref. 21). Moreover, in such a case the set of localized-magnon states in the subspaces $S^{z}=N s-n, n=2, \ldots, n_{\max }$ is linearly independent (theorem 5 of Ref. 21). Note that boundary conditions are crucial in this case. As was pointed out already in Ref. 24, there is indeed one relation between localized one-magnon states for the kagomé lattice if periodic boundary conditions are imposed. For the checkerboard lattice with periodic boundary conditions there is a similar linear relation [21]. By contrast, all localized one-magnon states are linearly independent if one considers the kagomé or checkerboard lattice with open boundary conditions. Note that for the pyrochlore lattice there is more than one linear relation between one-magnon states localized on hexagons and the linear relations between such localized-magnon states exist also in the subspaces $S^{z}=N s-n$ for $n>1$ [21].

The second important issue concerns the completeness of the localized-magnon states in each sector of $S^{z}=N s, \ldots, N s-n_{\max }$. More precisely, a quantitative description of the low-temperature thermodynamics of the spin model in the vicinity of the saturation field is obtained from the smallest-size localized magnons via Eq. (5) only if there are no further thermodynamically relevant contributions. Again we do not know a general answer. However, there are topological arguments supporting the existence of such additional contributions in certain higher-dimensional systems, and suggesting their absence otherwise. Furthermore, we can perform a quantitative comparison for each specific spin system separately with numerical results for finite systems.
From exact diagonalization data for finite systems with periodic boundary conditions we infer that for some of the lattices there are no additional states, or at most one or two additional states in the subspace $S^{z}=N s-1$. A perfect correspondence is found for the diamond chain and the frustrated two-leg ladder with $J_{2}>2 J_{1}$ as well as the sawtooth chain and certain two-dimensional models with finite localization regions such as the frustrated bilayer lattice with $J_{2}>4 J_{1}$ (see Ref. 20 and Table 1). In other cases such as the kagomé-like chains there are only one or two extra states which can be traced to one-magnon states from the dispersive band which at one or two values of the wave-vector have the same energy as the states from the flat band. In these cases which include in particular the one-dimensional models, a quantitatively accurate description is expected from the smallest-size localized magnons.

In contrast, the topology of certain two-dimensional lattices permits the construction of additional, nested localized-magnon states $[13,24]$. An examples of such a «defect» state is shown in Fig. 3,b. Such defect states occur first in the two-magnon sector and, once again, the issue of linear independence from the smallest-area localized magnon states arises. We would like to point out that boundary conditions again play a crucial role: for open boundary conditions, the two-magnon state obtained from the two nested loops at the lower left corner of Fig. $3, b$ is indeed a new state. However, for the periodic boundary conditions which we are considering here, such states can be expressed as a linear combination of smallest-area localized two-magnon states. Let us explain this in the case of the checkerboard lattice. First note that in a finite checkerboard lattice with periodic boundary conditions, a magnon state localized on a closed loop $L$ such as

Table 1 . The degeneracies and the energy gaps for various finite $s=1 / 2$ frustrated bilayer lattices: exact diagonalization data for finite systems with $J_{2}=5 J_{1}$ vs hard-square predictions. $N$ is the number of sites in the spin lattice, DGS is the degeneracy of the ground state as it follows from exact diagonalization for a given $S^{z}$, \# HSS is the number of configurations with $N / 2-S^{z}$ hard squares, $\Delta$ is the energy gap between the ground state and the first excited one in units of $J_{1}$. The dots for $N=64$ indicate omitted sectors with $16 \leq S^{z} \leq 26$.

\begin{tabular}{c|c|c|c|c||c|c|c|c|c||c|c|c|c|c}
\hline \hline$N$ & $S^{z}$ & DGS & $\Delta$ & \#HSS & $N$ & $S^{z}$ & DGS & $\Delta$ & \#HSS & $N$ & $S^{z}$ & DGS & $\Delta$ & \#HSS \\
\hline \hline \multirow{2}{*}{16} & 7 & 8 & 1.0 & 8 & 32 & 15 & 16 & 1.0 & 16 & 64 & 31 & 32 & 1.0 & 32 \\
& 6 & 12 & 1.0 & 12 & & 14 & 88 & 1.0 & 88 & & 30 & 432 & 1.0 & 432 \\
& 5 & 8 & 2.0 & 8 & & 13 & 208 & 1.0 & 208 & & 29 & 3232 & 1.0 & 3232 \\
& 4 & 2 & 3.0 & 2 & & 12 & 228 & 1.0 & 228 & & 28 & & 1.0 & 14840 \\
\hline \multirow{2}{*}{20} & 9 & 10 & 1.0 & 10 & & 11 & 128 & 1.0 & 128 & & 27 & & \\
& 8 & 25 & 1.0 & 25 & & 10 & & 1.0 & 56 & & & & \\
& 7 & 20 & 1.0 & 20 & & 9 & & 2.0 & 16 & & & & \\
& 6 & 10 & 2.0 & 10 & & 8 & & 3.0 & 2 & & & & \\
& 5 & 2 & 3.0 & 2 & & & & & & & & & \\
\hline \hline
\end{tabular}


the large loop in Fig. 3, $b$ can be written as a linear combination of all magnon states localized on the smallest squares contained in $L$. But this linear combination is not unique since the set of all smallest localized one-magnon states is not linearly independent. Another linear combination yielding the same state could run over all magnon states $|q\rangle$ localized on the smallest squares $q$ not contained in $L$. Consider in particular the octagonal loop $L$ containing a smallest square $q_{0}$ with a magnon state $\left|q_{0}\right\rangle$ in its center shown in Fig. 3,b. Then the state of the two magnons localized on $L$ and $q_{0}$ can be written as a linear combination of large-hard-square two-magnon states $|q\rangle\left|q_{0}\right\rangle$ where $|q\rangle$ runs over all one-magnon states of smallest area not contained in $L$.

Note that the derivation of the linear relation relies on the absence of obstacles such as open boundaries or other localized magnons outside the two-magnon defect state. We therefore believe that the majority of many-magnon states constructed with these defect states (in particular the three-magnon state of Fig. 3,b) is linearly independent of many-magnon states containing only those of smallest area. The many-defect states are therefore expected to yield another finite (even if small) contribution to the ground-state entropy at the saturation field. Indeed, numerical data for finite lattices exhibits a larger ground-state degeneracy than predicted from the effective hard-object description (see Table 2 for the kagomé lattice and Table 3 for the checkerboard lattice). This difference should remain relevant in the thermodynamic limit according to the preceding argument based on the defect states.
Before we proceed, we would like to comment on some specific sectors for the kagomé lattice (Table 2) and the checkerboard lattice (Table 3). Firstly, the degeneracy of the ground-state (DGS) in the one-magnon sector is $N / 3+1(N / 2+1)$ for the kagomé (checkerboard) lattice. There are two possible interpretations (see also [24]): in momentum space this corresponds to the $N / 3(N / 2)$ states of the flat branch plus one additional state where the next dispersive branch touches the flat branch; in real space this corresponds to $N / 3(N / 2)$ smallest localized-magnon states subject to one linear relation plus two additional states which wind once around the boundaries. Secondly, the exact degeneracy in the two-magnon sector is given by $N^{2} / 18-N / 2+1$ for the kagomé lattice and $N^{2} / 8-5 N / 4+1$ for the checkerboard lattice. The difference with respect to the number of smallest-area localized-magnon configurations is again due to states with non-trivial winding. Let us explain this briefly for the checkerboard lattice. First, there are $N$ configurations of the form $|q\rangle\left|w_{x}\right\rangle$ or $|q\rangle\left|w_{y}\right\rangle$ where $q$ runs over all squares and $w_{\alpha}$ is an arbitrary loop which winds around boundary $\alpha=x, y$ sufficiently far away from $q$. As we will explain in detail elsewhere, taking into account states $\left|w_{\alpha}\right\rangle\left|w_{\alpha}^{\prime}\right\rangle$ with double winding together with one special diagonal state and the linear relations between these states, one finds $N+1$ additional linearly independent two-magnon states with non-trivial winding, i.e., exactly the same number as observed numerically. The difference $2 N / 3+1$ between the number of configurations of two hard hexagons and the exact ground-state degeneracy in the two-magnon sector

Table 2. The degeneracies and the energy gaps for the $s=1 / 2$ kagomé lattice: exact diagonalization data for finite systems vs hard-hexagon predictions. $N$ is the number of sites in the spin lattice, DGS is the degeneracy of the ground state as it follows from exact diagonalization for a given $S^{z}$, \# HHS is the number of configurations with $N / 2-S^{z}$ hard hexagons, $\Delta$ is the energy gap between the ground state and the first excited one in units of $J$. Dots indicate some sectors with $S^{z} \geq 7 N / 18$ which have been omitted for larger values of $N$. For the two-magnon sector one has \# HHS $=N^{2} / 18-7 N / 6$ and DGS $=N^{2} / 18-N / 2+1$.

\begin{tabular}{|c|c|c|c|c|c|c|c|c|c|c|c|c|c|c|}
\hline$N$ & $S^{z}$ & DGS & $\Delta$ & \#HHS & $N$ & $S^{z}$ & DGS & $\Delta$ & \#HHS & $N$ & $S^{z}$ & DGS & $\Delta$ & \#HHS \\
\hline \multirow[t]{4}{*}{36} & 17 & 13 & 0.500 & 12 & \multirow[t]{6}{*}{54} & 26 & 19 & 0.177 & 18 & \multirow[t]{3}{*}{108} & 53 & 37 & 0.177 & 36 \\
\hline & 16 & 55 & 0.182 & 30 & & 25 & 136 & 0.091 & 99 & & 52 & 595 & 0.095 & 522 \\
\hline & 15 & 71 & 0.055 & 16 & & 24 & 430 & 0.025 & 180 & & & & & $\ldots$ \\
\hline & 14 & 8 & 0.034 & 3 & & 23 & 513 & 0.009 & 99 & 192 & 95 & 65 & 0.101 & 64 \\
\hline \multirow[t]{5}{*}{45} & $43 / 2$ & 16 & 0.251 & 15 & & 22 & 119 & 0.003 & 18 & & 94 & 1953 & 0.067 & 1824 \\
\hline & $41 / 2$ & 91 & 0.123 & 60 & & 21 & 4 & 0.012 & 3 & & & & & $\ldots$ \\
\hline & $39 / 2$ & 201 & 0.035 & 60 & \multirow[t]{3}{*}{63} & $61 / 2$ & 22 & 0.297 & 21 & & & & & \\
\hline & $37 / 2$ & 110 & 0.011 & 15 & & $59 / 2$ & 190 & 0.128 & 147 & & & & & \\
\hline & $35 / 2$ & 4 & 0.012 & 3 & & $57 / 2$ & 785 & 0.050 & 406 & & & & & \\
\hline
\end{tabular}


Table 3. The degeneracies and the energy gaps for various finite $s=1 / 2$ checkerboard lattices: exact diagonalization data for finite systems vs large-hard-square predictions. $N$ is the number of sites in the spin lattice, DGS is the degeneracy of the ground state as it follows from exact diagonalization for a given $S^{z}$, \# LHSS is the number of configurations with $N / 2-S^{z}$ large-hard-squares, $\Delta$ is the energy gap between the ground state and the first excited one in units of $J$. Dots indicate some sectors with $S^{z} \geq 3 N / 8$ which have been omitted for larger values of $N$. For the two-magnon sector one has \# LHSS $=N^{2} / 8-9 N / 4$ and DGS $=N^{2} / 8-5 N / 4+1$.

\begin{tabular}{|c|c|c|c|c|c|c|c|c|c|c|c|c|c|c|}
\hline$N$ & $S^{z}$ & DGS & $\Delta$ & \#LHSS & $N$ & $S^{z}$ & DGS & $\Delta$ & \#LHSS & $N$ & $S^{z}$ & DGS & $\Delta$ & \#LHSS \\
\hline \multirow[t]{8}{*}{40} & 19 & 21 & 0.882 & 20 & \multirow[t]{8}{*}{64} & 31 & 33 & 0.586 & 32 & \multirow[t]{4}{*}{144} & 71 & 73 & 0.268 & 72 \\
\hline & 18 & 151 & 0.222 & 110 & & 30 & 433 & 0.176 & 368 & & 70 & 2413 & 0.128 & 2268 \\
\hline & 17 & 411 & 0.071 & 180 & & 29 & 2833 & 0.082 & 1888 & & 69 & & & 41208 \\
\hline & 16 & 246 & 0.014 & 85 & & 28 & \multirow[t]{5}{*}{9273} & \multirow[t]{5}{*}{0.026} & 4392 & & & & & $\ldots$ \\
\hline & 15 & 4 & 0.028 & 4 & & 27 & & & 4224 & \multirow[t]{4}{*}{256} & 127 & 129 & 0.152 & 128 \\
\hline & & & & & & 26 & & & 1520 & & 126 & 7873 & 0.098 & 7616 \\
\hline & & & & & & 25 & & & 224 & & 125 & & & 279936 \\
\hline & & & & & & 24 & & & 12 & & & & & \\
\hline
\end{tabular}

for the kagomé lattice can be explained in an analogous way. Finally, let us look at the sector with the closest packing of localized magnons, i.e., $S^{z}=7 N / 18$ for the kagomé lattice and $S^{z}=3 N / 8$ for the checkerboard lattice. For the $N=40$ checkerboard lattice we find the same number of ground states and large-hard-square states for $S^{z}=15$, as expected for a closest packing. By sharp contrast, the kagomé lattice gives rise to 8 ground states for $N=36$, and 4 for $N=45,54$ in the sector with $S^{z}=7 N / 18$ while one expects only 3 for the magnon crystal $[3,8,24]$. Since the $N=36,45$ and 54 kagomé lattices should be sufficiently large to eliminate boundary artifacts, the origin of the additional state(s) is unclear at present. In particular, it remains to be clarified whether (essentially) all ground states are described by localized magnons if all topological non-trivial configurations (including defect states and states with non-trivial winding) are properly accounted for.

One further issue is whether the ground-state manifold is separated from other states by a finite energy gap. We can again draw some conclusions concerning the energy gap from exact diagonalization data. In previous papers $[20,22]$ we introduced a measure for the thermodynamically relevant energy separation $\Delta_{D O S}$ between the ground-state manifold and the other eigenstates of the system. For simplicity, in the present paper we report the energy gap $\Delta$ between the ground-state energy and the next smallest energy level in each sector $S^{z}$. Tables 1,2 , and 3 present the values of $\Delta$ for some finite frustrated bilayer, kagomé, and checkerboard lattices, respectively. For the frustrated bilayer lattice with $J_{2}=5 J_{1}$, the localized magnons are separated from all higher excitations by an energy gap $\Delta \geq J_{1}$ (see Table 1 ). For the kagomé lattice one has the additional complication that there is no gap to the next branch of excitations in the one-magnon sector. Nevertheless, two-magnon scattering states were estimated to have an energy gap $\Delta \approx 0.24 J[13,24]$. While this may be a valid estimate in the two-magnon sector of the kagomé lattice, in higher sectors there are definitely excitations at substantially lower energies (see Table 2). In fact, analysis of further excited states (not shown here) indicates the onset of a thermodynamically relevant density of states at energies of the order of only $10^{-2} \mathrm{~J}$.

\section{Low-temperature strong-field thermodynamics. Lattice-gas description}

As mentioned above, the smallest-area localized-magnon states may dominate the low-temperature thermodynamics in the vicinity of the saturation field. After having checked their linear independence we would like to discuss their contribution to the canonical partition function of the spin system in more detail. We start from Eq. (5), where this contribution is given. We emphasize once again that this formula describes the low-temperature thermodynamics near the saturation field accurately provided that (i) there are no other ground states (apart from the smallest-area localized-magnon states) in the corresponding sectors of $S^{z}$ or that the contribution of such extra states is vanishingly small as $N \rightarrow \infty$, and that (ii) excited states in these sectors are separated by a finite energy gap from the ground states. In Eq. (5) $g_{N}(n)$ is the degeneracy of localized states of $n$ isolated smallest-area magnons on a spin lattice of $N$ sites. It is useful to consider $g_{N}(n)$ as the canonical partition function $Z(n, \mathcal{N})$ of $n$ hard-core objects on an auxiliary lattice of $\mathcal{N} \propto N$ sites $(\mathcal{N}=N / 3, N / 2, N / 3$, and $N / 2$ for the diamond chain, the frustrated two-leg ladder, the kagomé-like chain, and the 
frustrated bilayer lattice, respectively). We can write the grand-canonical partition function of hard-core objects on this lattice as

$$
\Xi(T, \mu, \mathcal{N})=\sum_{n=0}^{n_{\max }} g_{N}(n) \exp (\mu n / k T),
$$

where $\mu$ is the chemical potential of the hard-core objects. The simple reason why a hard-core object lattice-gas description emerges here is the existence of the «hard-core rules» which the localized-magnon states must respect in order to be eigenstates of the spin Hamiltonian. Note that these rules differ for various spin lattices: the diamond chain of Fig. 1, $a$ gives rise to hard monomers, the frustrated ladder (Fig. 1,b) and the kagomé-like chain (Fig. $1, c)$ are described by hard dimers, while the hard-core objects for two-dimensional lattices are illustrated in Fig. 2. Using $\Xi(T, \mu, \mathcal{N})$ we arrive at the basic relation between the localized-magnon contribution to the canonical partition function of the spin model and the grand-canonical partition function of the corresponding hard-core object lattice-gas model,

$$
Z_{l m}(T, h, N)=\exp \left(-\frac{E_{F M}-h N s}{k T}\right) \Xi(T, \mu, \mathcal{N}),
$$

with $\mu=h_{1}-h$. From Eq. (6) we find the Helmholtz free energy of the spin system

$$
\frac{F_{l m}(T, h, N)}{N}=\frac{E_{F M}}{N}-h s-k T \frac{\mathcal{N}}{N} \frac{\ln \Xi(T, \mu, \mathcal{N})}{\mathcal{N}} .
$$

The entropy $S$, the specific heat $C$, the magnetization $M=\left\langle S^{z}\right\rangle$, and the susceptibility $\chi$ follow from (7) according to usual formulae,

$$
\begin{gathered}
S_{l m}(T, h, N)=-\partial F_{l m}(T, h, N) / \partial T, \\
C_{l m}(T, h, N)=T \partial S_{l m}(T, h, N) / \partial T, \\
M_{l m}(T, h, N)=N s-k T \partial \ln \Xi(T, \mu, \mathcal{N}) / \partial \mu, \text { and } \\
\chi_{l m}(T, h, N)=\partial M_{l m}(T, h, N) / \partial h,
\end{gathered}
$$

respectively. Note that the thermodynamic quantities of the spin system depend on the temperature $T$ and the magnetic field $h$ essentially only through the combination $x=\left(h_{1}-h\right) / k T[20]$.

To test the hard-object description, we have performed full diagonalization of spin-1/2 isotropic Heisenberg systems (i.e., $\Delta=1$ in Eq. (1)), imposing periodic boundary conditions. For the diamond chain and the frustrated ladder we have also exploited the local conservation laws. First we rewrite the Hamiltonian in terms of the total spin on the vertical dimers in Fig. 1 [32]. For a local spin 1/2, each dimer can only be in the singlet or triplet state. A singlet cuts the system into smaller fragments. It is therefore sufficient to compute the spectra of one periodic fragment where all vertical dimers are in the triplet state, and smaller open fragments where all consecutive dimers are again in the triplet state. In this manner it requires only a moderate effort to obtain the complete spectra for a diamond chain and a frustrated ladder with $N=24$, while $N=24$ would be inaccessible for the diamond chain with a full diagonalization of the original model.

\subsection{Hard-monomer universality class}

First we consider the hard-monomer universality class which includes the diamond chain, the dimer-plaquette chain and the square-kagomé lattice. The hard-monomer restriction for these lattices means that it is forbidden to have two (or more) localized magnons in the same trap. We focus on the diamond chain (see Fig. 1,a), and refer the interested reader to Ref. 20 for the other lattices. A straightforward calculation yields the grand-canonical partition function of a gas of hard monomers

$$
\Xi(T, \mu, \mathcal{N})=\left(1+\exp \frac{\mu}{k T}\right)^{\mathcal{N}} .
$$

Explicit analytic expressions for thermodynamic quantities can be obtained easily [20]. Note that the thermodynamic quantities per site are independent of the system size $N$. At the saturation field we have a residual ground-state entropy $S_{l m}\left(T, h_{1}, N\right) / k N=(\mathcal{N} / N) \ln 2$. The specific heat $C_{l m}\left(T, h_{1}, N\right) / k N$ exhibits two identical maxima of height $\approx 0.43922884 \mathcal{N} / N$ at $x \approx \pm 2.39935728$.

Some typical dependencies of the thermodynamic quantities on the field and the temperature for the diamond chain with $J_{1}=1, J_{2}=3$ and the corresponding hard-monomer data obtained on the basis of Eq. (8) are shown in Fig. 4, left column. Deviations between exact diagonalization (ED) and hard monomers (HM) are observed only in the specific heat $C$, and only for $k T \gtrsim 0.2 J_{1}$ (lowest panel in the left column of Fig. 4). Note furthermore that thermodynamic quantities are symmetric under $x \rightarrow-x$ within the hard-monomer picture. In particular, the hard-monomer description yields a susceptibility $\chi$ and a specific heat $C$ for the diamond chain which coincide at $h=3.8 J_{1}$ and $h=4.2 J_{1}$.

\subsection{One-dimensional hard-dimer universality class}

The frustrated two-leg ladder, the kagomé-like chains, and the sawtooth chain belong to the one-dimensional hard-dimer universality class, i.e., the rules for the localized magnons obey the restrictions for rigid dimers on a one-dimensional lattice: each trapping cell can only be occupied by one localized magnon and neighboring trapping cells cannot be simultaneously occupied by localized magnons. We focus on the frustrated two-leg ladder and the kagomé-like chain of type I (see Figs. 1,b,c). The grand-canonical partition function of one-dimensional 

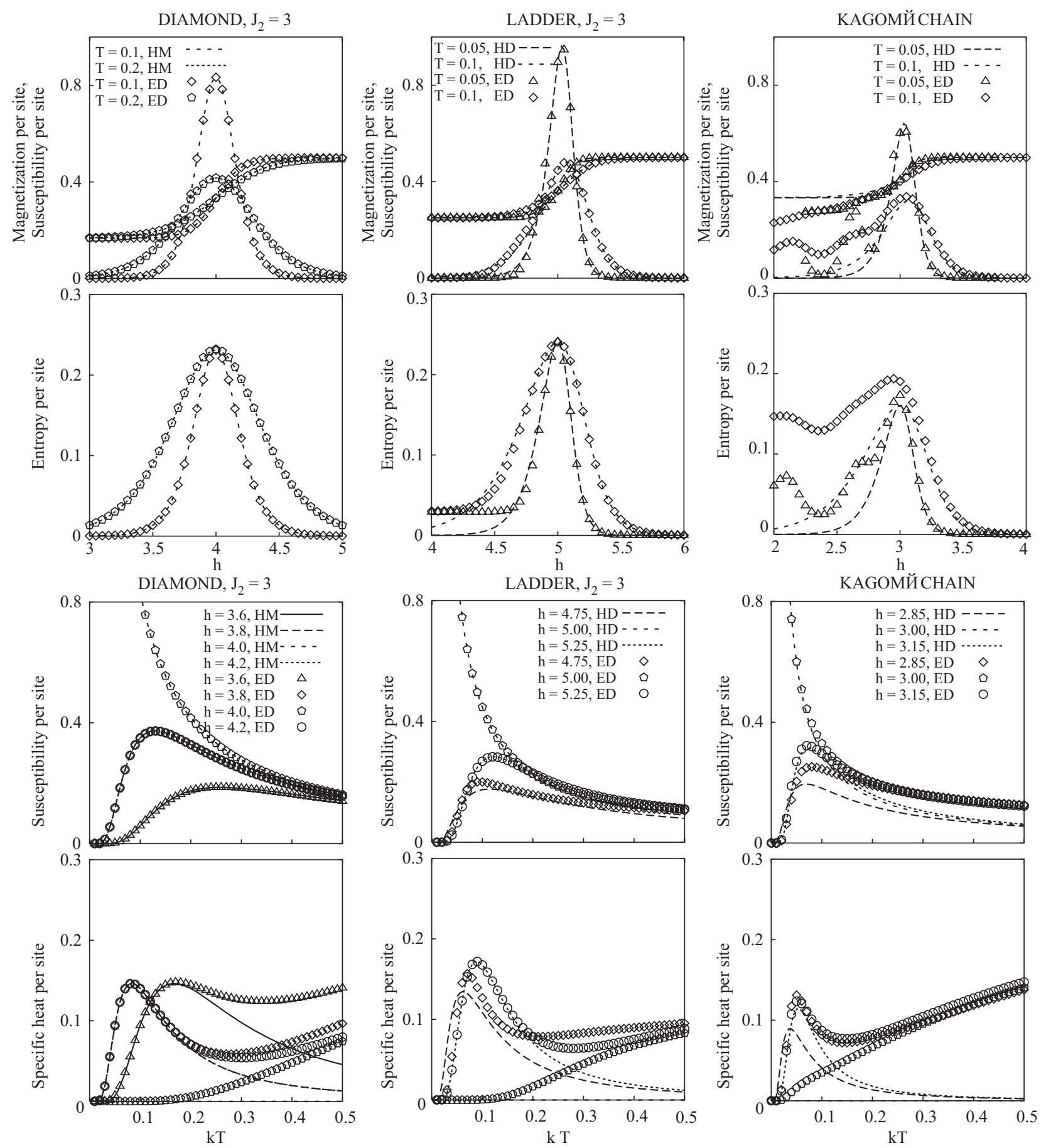

Fig. 4. $M(T, h, N) / N, \chi(T, h, N) / N$, and $S(T, h, N) / k N$ vs $h$ at low temperatures; $\chi(T, h, N) / N$ and $C(T, h, N) / k N$ vs $k T$ around the saturation field. From left to right: diamond chain with $J_{1}=1, J_{2}=3$, frustrated two-leg ladder with $J_{1}=1, J_{2}=3$, kagomé-like chain with $J=1$. We set the field range $h_{1}-1 \leq h \leq h_{1}+1$ and the temperature range $0 \leq k T \leq 0.5$. The exact diagonalization (ED) data (symbols) refer to finite systems of sizes $N=24$ (diamond chain and frustrated ladder) and $N=18$ (kagomé-like chain). The analytical predictions for hard monomers $(\mathrm{HM})$ and one-dimensional hard dimers $(\mathrm{HD})(\mathcal{N} \rightarrow \infty)$ are shown by lines.

hard dimers can be calculated with the help of the transfer-matrix method:

$$
\Xi(T, \mu, \mathcal{N})=\lambda_{1}^{\mathcal{N}}+\lambda_{2}^{\mathcal{N}}, \quad \lambda_{1,2}=\frac{1}{2} \pm \sqrt{\frac{1}{4}+\exp \frac{\mu}{k T}}
$$

Explicit analytic expressions for thermodynamic quantities can again be obtained easily. Note that for hard dimers the thermodynamic quantities per site depend on the size $\mathcal{N}$. In the thermodynamic limit only the largest eigenvalue $\lambda_{1}$ plays a role. Explicit expressions in this limit can be found in Refs. 12,24 for the sawtooth chain 
and in Ref. 20 for the general case. At the saturation field one finds a residual ground-state entropy

$$
S_{l m}\left(T, h_{1}, N\right) / k N=(\mathcal{N} / N) \ln ((1+\sqrt{5}) / 2) .
$$

The specific heat $C_{l m}\left(T, h_{1}, N\right) / k N$ exhibits two maxima of height $\approx 0.34394234 \mathcal{N} / N$ (at $x \approx-2.81588498$ ) and $\approx 0.26887020 \mathcal{N} / N$ (at $x \approx 4.05258891$ ). Some typical dependencies of the thermodynamic quantities on field and temperature are shown in Fig. 4, middle and right columns for the frustrated ladder with $J_{1}=1, J_{2}=3$ and the kagomé-like chain with $J=1$, respectively. Note that the hard-dimer results are not symmetric around the saturation field, i.e., all thermodynamic quantities are different for $x$ and $-x$.

For the frustrated ladder with $J_{2}=3 J_{1}$, we observe again systematic differences between exact diagonalization and hard dimers in the specific heat $C$ at high temperatures (lowest panel in the middle column of Fig. 4). The remaining differences for low temperatures and $h<h_{1}$ are due to finite-size effects, since in Fig. 4 we compare finite spin systems with infinite hard-dimer systems. If the comparison is performed for the same system size [20], better agreement can be observed. One important source of finite-size effects is a two-fold degeneracy of the ground state at $M / N=1 / 4$. This ground-state degeneracy is evident in the finite value of the ED results for the entropy $S$ at the left side of the second panel in the middle column of Fig. 4.

In the case of the kagomé-like chain (right column of Fig. 4) we find good agreement between exact diagonalization and hard dimers on the high-field side $h \geq h_{1}$ and sufficiently low temperatures. However, on the low-field side $h<h_{1}$ we observe even stronger deviations than for the frustrated ladder. Indeed, there are two steps at $h_{2} \approx 2.67 \mathrm{~J}$ and $h_{3} \approx 2.11 \mathrm{~J}$ in the zero-temperature magnetization curve of the $N=18$ kagomé chain with $M / N<1 / 3$ (see also Fig. 2 of Ref. 23). Since the region $M / N<1 / 3$ cannot be described with hard dimers, the region with $h \lesssim 2.7 \mathrm{~J}$ falls completely outside the validity of the hard-dimer picture.

\subsection{Two-dimensional lattice gases, hard-square universality class}

The frustrated bilayer lattice, the kagomé lattice as well as the checkerboard lattice are even more interesting, since the corresponding two-dimensional classical hard-core systems exhibit a second-order finite-temperature order-disorder phase transition [40-42]. However, recall from Sec. 3 that the spin model exhibits extra ground states on the kagomé and checkerboard lattices which cannot be described by hard hexagons or large-hard-squares, respectively. Moreover, the gap to excited states is pretty small, see Tables 2 and 3. Therefore, the description of the kagomé and checkerboard lattices in terms of hard-core objects is expected to be only a qualitative one.

We therefore focus on the frustrated bilayer antiferromagnet as an example for a finite-temperature order-disorder phase transition [22]. In this case, exact diagonalization provides clear evidence that all ground states are mapped onto the hard-square configurations on a square lattice, see Table 1. Moreover, the gap to excited states is large. Thus, the low-temperature strong-field thermodynamics should be determined completely by the hard-square problem. Although we do not know the exact analytical result for the grand thermodynamical potential $-k T \ln \Xi(T, \mu, \mathcal{N}) / \mathcal{N}$ of hard squares on a square lattice, the properties of the model are well known [40,42]. In particular, the hard-square model exhibits a phase transition at $z_{c}=\exp \left(\mu_{c} / k T\right)=3.7962 \ldots$ between the low-density phase $\left(z<z_{c}\right)$, in which both sublattices of the underlying square lattice are equally occupied, and the high-density phase $\left(z>z_{c}\right)$, in which one of the sublattices becomes more occupied than the other one. In spin language, the phase transition has a purely geometrical origin and indicates the ordering of localized-magnon states as their density varies with field or temperature. The phase transition belongs to the two-dimensional Ising universality class. Hence, the specific heat should show a logarithmic singularity at the critical point. Fig. 5 shows results for the temperature dependence of the specific heat around the saturation field for the frustrated bilayer lattice. The available exact diagonalization data are restricted to rather small spin systems. However, they demonstrate a perfect agreement with the results for the corresponding finite hard-square model: for $J_{2}=5 J_{1}$ and $h=0.99 h_{1}=8.91 J_{1}$ or $h=1.01 h_{1}=9.09 J_{1}$, both data sets in Fig. 5 are indistinguishable for temperatures $k T \lesssim 0.1 J_{1}$. Bigger hard-square systems can be studied using classical Monte Carlo simulations [43]. The left panel of Fig. 5 shows that a logarithmic singularity in the dependence of $C$ vs $T$ develops on the low-field side with increasing size of the hard-square model. Since this transition occurs within the temperature region where the exact diagonalization data are perfectly reproduced by the hard-square model, we expect that this singularity also appears in the spin model in the thermodynamic limit $N \rightarrow \infty$.

To summarize this subsection, the frustrated bilayer lattice provides an example where a two-dimensional hard-core lattice gas completely covers all low-energy states of the spin model. In this model, there is a clear phase transition which corresponds to a crystallization of hard squares, i.e., magnons localized on the vertical dimers. We would like to emphasize that such a phase transition does not contradict the Mermin-Wagner theorem [44] since only a discrete symmetry is broken spontaneously. This demonstration of a finite-temperature phase transition in a two-dimensional interacting many-body 

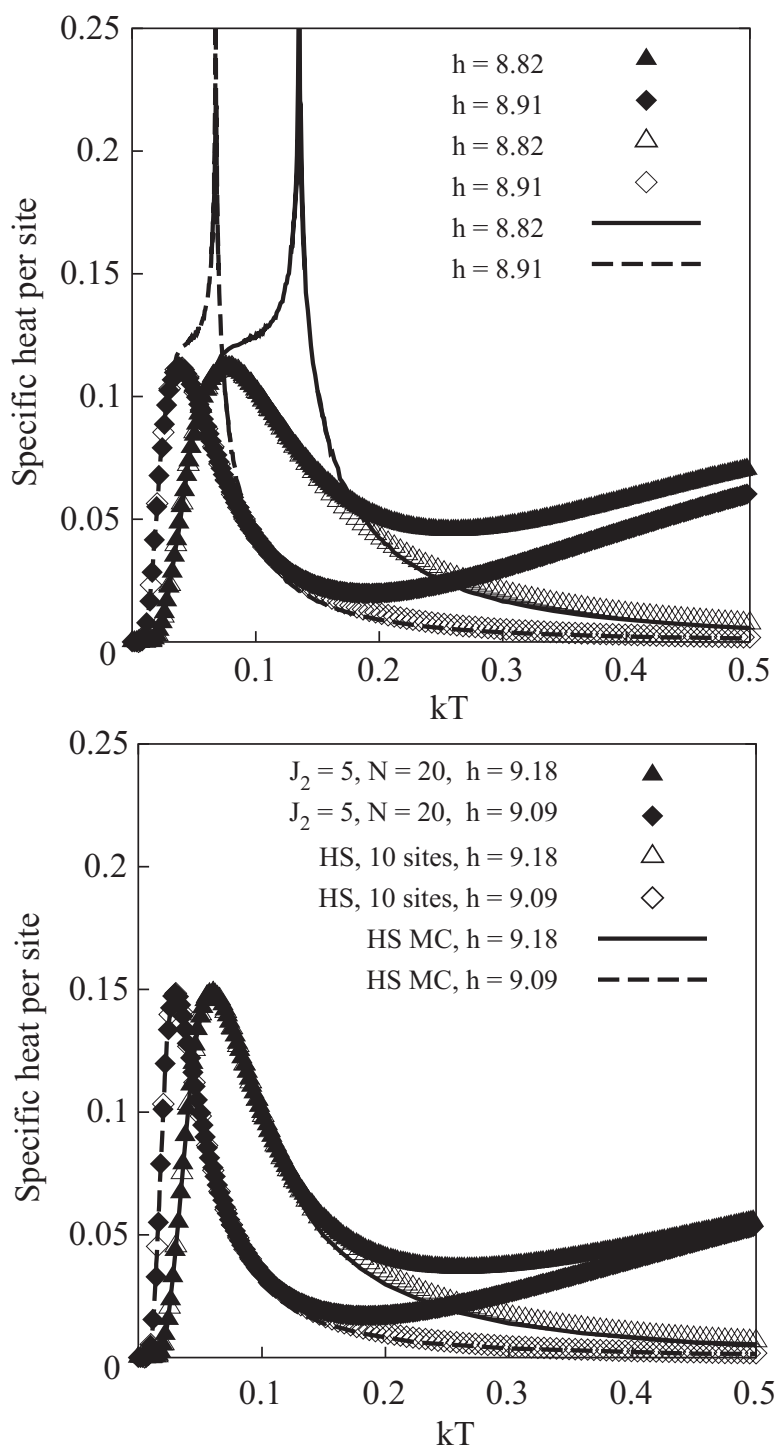

Fig 5. $C(T, h, N) / k N$ vs $k T$ around the saturation field $h_{1}=9$ for the frustrated bilayer lattice with $J_{1}=1, J_{2}=5$. The exact diagonalization data (filled symbols) refer to a finite spin system of $N=20$ sites. The analytical results (empty symbols) refer to a finite hard-square system of $\mathcal{N}=10$ sites. The Monte Carlo simulation data (lines) are obtained for the hard-square system on finite lattices with $\mathcal{N}$ up to $800 \times 800$.

spin model is an interesting example for the impact of the localized-magnon states on the physical properties of a wide class of frustrated magnets.

\subsection{Region of validity}

Finally, we add some general remarks about the region of validity of the hard-core lattice-gas description. Obviously, this effective picture of the spin model is accurate only in some region in the $(h-T)$-plane around the point $h=h_{1}, T=0$. The field $h<h_{1}$, until which the hard-core object picture should work at $T=0$, is related to the width of the plateau $h_{1}-h_{2}$ preceding the jump in the ground-state magnetization curve $[8,10,11,23]$ with $h_{2}$ being the dif- ference between the ground-state energy in the sectors $S^{z}=N s-n_{\max }$ and $S^{z}=N s-n_{\max }-1$. We could also try to estimate a characteristic temperature $T^{*}(h)$, below which the hard-core object picture should work at a certain magnetic field $h$. At $h=h_{1}$ we can find $T^{*}$ from the temperature dependence of the specific heat $C$. Indeed, the hard-core object prediction is $C=0$ for all temperatures at $h=h_{1}$. However, as can be seen in the corresponding panels in Fig. 4 this is a valid approximation for $k T \lesssim$ $0.2 J_{1}\left(k T \lesssim 0.15 J_{1}\right)$ for the diamond chain (frustrated ladder), whereas the corresponding temperature region is much smaller for the kagomé-like chain. For $h>h_{1}$ the temperature $T^{*}$ depends on the difference $h-h_{1}$. Numerical data for finite systems indicates that $T^{*}$ increases with growing $h-h_{1}$.

Lastly, we note that a description of the low-energy degrees of the spin systems can be extended by relaxing the hard-core rules, e.g., by rendering the infinite nearest-neighbor repulsion finite or permitting double occupation of the auxiliary lattice sites [20,24]. In such a case we may achieve a better agreement with exact diagonalization data in a wider range of parameters around the point $h=h_{1}, T=0$, loosing, however, the universal dependence on $h$ and $T$ only via the parameter $x=\left(h_{1}-h\right) / k T$.

\section{Conclusions}

In this paper, we have discussed the universal properties of some highly frustrated quantum Heisenberg antiferromagnets supporting localized-magnon eigenstates. Universal behavior emerges owing to the localized-magnon states which become the ground states around the saturation field and can be separated from the higher-energy states by an energy gap. We find several universality classes depending on the specific lattice-gas model of hard-core objects which describes the low-energy degrees of freedom of the spin model in strong magnetic fields. For the one-dimensional models, the lattice gas yields a quantitative description of the thermodynamics of the full spin model close to the saturation field and at sufficiently low temperatures.

Higher dimensions may be even more interesting since they allow for a finite-temperature crystallization phase transition of the hard-core objects. As a two-dimensional example, we have focused on the hard-square universality class which contains, e.g., the frustrated bilayer quantum Heisenberg antiferromagnet in the region with sufficiently strong interlayer coupling [22]. Such a spin model exhibits an order-disorder phase transition of a purely geometrical origin which reflects the geometrical ordering of the localized magnons. It turns out that the phase transition of hard squares belongs to the two-dimensional Ising universality class and is characterized by a logarithmic singularity of the specific heat just below the saturation field. 
New numerical results and complementary arguments presented in this paper indicate that the situation is more complicated in the case of the kagomé and checkerboard lattices. Here, there seem to be thermodynamically relevant contributions to the ground-state manifold beyond that of hard hexagons and large-hard squares, respectively. Accordingly, in these cases the gas of smallest hard objects cannot be expected to yield a quantitatively accurate description of the spin model in any parameter regime. Still, the universality class of a possible crystallization phase transition should be predicted correctly by such an effective low-energy theory.

We believe that the properties of localized magnons elucidate the physics of frustrated quantum antiferromagnets in high magnetic fields and thus are useful for a general understanding of related compounds. Even more, recent experiments on the spin-1/2 (distorted) diamond-chain compound azurite $\mathrm{Cu}_{3}\left(\mathrm{CO}_{3}\right)_{2}(\mathrm{OH})_{2}[26,27]$ and the frustrated quasi-two-dimensional spin-1/2 antiferromagnet $\mathrm{Cs}_{2} \mathrm{CuCl}_{4}$ [28] raise hopes for a direct comparison with the theoretical models discussed in this paper, although so far there is no clear experimental observation of the pronounced quantum effects predicted for low-dimensional spin-1/2 antiferromagnets with localized magnons yet.

The $\mathrm{Cu}^{2+}$ ions in azurite $\mathrm{Cu}_{3}\left(\mathrm{CO}_{3}\right)_{2}(\mathrm{OH})_{2}$ form infinite chains with the structure of a spin-1/2 distorted diamond chain [26,27]. The high-field magnetization $M(h)$ of azurite exhibits a plateau at $1 / 3$ of the saturation magnetization and a further steep increase as the magnetic field tends to the saturation value of about $32.5 \mathrm{~T}[26,27]$. Note that an experimentally accessible saturation field is an attractive feature of this compound. Fits of the magnetization curve and thermodynamic properties with high-temperature series [37] and numerical results yield the following estimates for the exchange interactions [27]: $J_{1}^{w}=8.6 \mathrm{~K}, J_{1}^{S}=19 \mathrm{~K}, J_{2}=24 \mathrm{~K}$ (in Fig. 1, $a$ the bonds with weaker interaction $J_{1}^{w}$ run from south-west to north-east whereas the bonds with stronger interaction $J_{1}^{S}$ run from north-west to south-east). Deviations from the ideal diamond chain geometry are not necessarily a major problem (see below). However, the quoted estimates for the exchange interactions are not in the region $J_{2} \geq 2 J_{1}$ which is required to render the localized magnons low-energy excitations (compare also the phase diagram of the distorted diamond chain [45]). Nevertheless, the values of the exchange couplings are still under debate [27].

$\mathrm{Cs}_{2} \mathrm{CuCl}_{4}$ is a frustrated quasi-two-dimensional spin- $1 / 2$ antiferromagnet with a low saturation field of only about $8.5 \mathrm{~T}$ [46]. For this compound, measurements of the low-temperature behavior of the specific heat around the saturation field have already been performed, exhibiting a strong dependence on the magnetic field [28]. The dominant exchange interactions in $\mathrm{Cs}_{2} \mathrm{CuCl}_{4}$ correspond to an anisotropic triangular lattice with strong interactions along one «chain» direction. For such a model one can construct magnons localized on the strongly coupled chains, much in the same way as for the frustrated square lattice $[3,8]$. Due to the quantization of momenta transverse to the chain direction, these localized magnon states turn out to be high-field ground states for up to at least six coupled chains in the parameter regime relevant to $\mathrm{Cs}_{2} \mathrm{CuCl}_{4}$ [46]. The localized magnons cease to be ground states as one approaches the thermodynamic limit, but they remain low-energy excitations. However, even in a case such as the frustrated square lattice where magnons localized on lines are high-field ground states for all finite systems, these are not thermodynamically relevant, but instead magnetic order occurs below the saturation field [47]. Indeed, $\mathrm{Cs}_{2} \mathrm{CuCl}_{4}$ exhibits a finite-temperature magnetic ordering transition below the saturation field [46] such that inter-plane coupling will have to be taken into account for a quantitative description of the low-temperature specific heat [28]. Nevertheless, the existence of localized magnons and the strong field-dependence of the specific heat of $\mathrm{Cs}_{2} \mathrm{CuCl}_{4}$ [28] are both related to the strong frustration of the anisotropic triangular lattice.

With respect to experiments such as those on azurite it is desirable to be not restricted to the «ideal geometry» allowing existence of exact localized-magnon ground states and to examine the «stability» of our results against small deviations from the relation which we have imposed on the exchange interactions. Numerical studies for finite spin systems [14] suggest that the main features coming from the localized-magnon states survive in case of small deviations from the ideal lattice geometry. In general, we can argue that due to small deviations from the ideal geometry, the flat magnon band becomes slightly dispersive but the hard-core constraint is preserved. Apparently, in this case we are faced with a quantum hard-core object model (e.g., the quantum hard-square model studied in Ref. 48, see also references therein). A study of the corresponding low-energy theories is beyond the scope of the present paper.

O.D. and J.R. acknowledge the kind hospitality of the MPIPKS-Dresden in the end of 2006. O.D. is indebted to Magdeburg University and to Wroclaw University for their hospitality in the autumn of 2006. A part of the numerical calculations was performed using Jörg Schulenburg's spinpack. We would like to thank M.E. Zhitomirsky for useful discussions and comments on this manuscript.

Note added. Two closely related preprints [49,50] were submitted shortly after the present one [51]. Ref. 49 confirms some of the new conclusions of our Sec. 3. Furthermore, Refs. 49,50 both contain Monte-Carlo results for the phase transition in the large-hard-square lattice 
gas. They agree on the location of the critical point while the universality class of the phase transition remains under debate.

1. C. Lhuillier and G. Misguich, in: High Magnetic Fields: Applications in Condensed Matter Physics and Spectroscopy, C. Berthier, L.P. Lévy, and G. Martinez (eds.), Springer, Berlin (2002), p. 161; G. Misguich and C. Lhuillier, in: Frustrated Spin Systems, H.T. Diep (ed.), World Scientific, Singapore (2005), p. 229.

2. H.-J. Mikeska and A. K. Kolezhuk, in: Quantum Magnetism, U. Schollwöck, J. Richter, D.J.J. Farnell, and R.F. Bishop (eds.), Springer, Berlin (2004), p. 1.

3. J. Richter, J. Schulenburg, and A. Honecker, in: Quantum Magnetism, U. Schollwöck, J. Richter, D.J J. Farnell, and R.F. Bishop (eds.), Springer, Berlin (2004), p. 85.

4. P. Lemmens and P. Millet, in: Quantum Magnetism, U. Schollwöck, J. Richter, D.J.J. Farnell, and R.F. Bishop (eds.), Springer, Berlin (2004), p. 433.

5. K. Penc, N. Shannon, and H. Shiba, Phys. Rev. Lett. 93, 197203 (2004); K. Penc, N. Shannon, Y. Motome, and H. Shiba, J. Phys.: Condens. Matter 19, 145267 (2007).

6. D.L. Bergman, R. Shindou, G.A. Fiete, and L. Balents, Phys. Rev. B74, 134409 (2006); D.L. Bergman, R. Shindou, G.A. Fiete, and L. Balents, Phys. Rev. 75, 094403 (2007).

7. J. Schnack, H.-J. Schmidt, J. Richter, and J. Schulenburg, Eur. Phys. J. B24, 475 (2001).

8. J. Schulenburg, A. Honecker, J. Schnack, J. Richter, and H.-J. Schmidt, Phys. Rev. Lett. 88, 167207 (2002).

9. H.-J. Schmidt, J. Phys. A35, 6545 (2002).

10. J. Richter, J. Schulenburg, A. Honecker, J. Schnack, and H.-J. Schmidt, J. Phys.: Condensed Matter 16, S779 (2004).

11. J. Richter, O. Derzhko, and J. Schulenburg, Phys. Rev. Lett. 93, 107206 (2004).

12. M.E. Zhitomirsky and A. Honecker, J. Stat. Mech.: Theor. Exp., P07012 (2004).

13. M.E. Zhitomirsky and H. Tsunetsugu, Phys. Rev. B70, 100403(R) (2004).

14. O. Derzhko and J. Richter, Phys. Rev. B70, 104415 (2004).

15. J. Richter, J. Schulenburg, A. Honecker, and D. Schmalfuß, Phys. Rev. B70, 174454 (2004).

16. J. Richter, J. Schulenburg, P. Tomczak, and D. Schmalfu $\beta$, arXiv:cond-mat/0411673.

17. R. Schmidt, J. Richter, and J. Schnack, J. Magn. Magn. Mater. 295, 164 (2005).

18. O. Derzhko and J. Richter, Phys. Rev. B72, 094437 (2005).

19. O. Derzhko, J. Richter, and J. Schulenburg, Phys. Status Solidi B242, 3189 (2005).

20. O. Derzhko and J. Richter, Eur. Phys. J. B52, 23 (2006).

21. H.-J. Schmidt, J. Richter, and R. Moessner, J. Phys. A39, 10673 (2006).

22. J. Richter, O. Derzhko, and T. Krokhmalskii, Phys. Rev. B74, 144430 (2006).

23. J. Schnack, H.-J. Schmidt, A. Honecker, J. Schulenburg, and J. Richter, J. Phys.: Conf. Ser. 51, 43 (2006).

24. M. E. Zhitomirsky and H. Tsunetsugu, Prog. Theor. Phys. Suppl. 160, 361 (2005).

25. J. Richter, Fiz. Nizk. Temp. 31, 918 (2005) [Low Temp. Phys. 31, 695 (2005)].
26. H. Kikuchi, Y. Fujii, M. Chiba, S. Mitsudo, and T. Idehara, Physica B329-333, 967 (2003); H. Kikuchi, Y. Fujii, M. Chiba, S. Mitsudo, T. Idehara, and T. Kuwai, J. Magn. Magn. Mater. 272-276, 900 (2004); S. Okubo, T. Kamikawa, T. Kunimoto, Y. Inagaki, H. Ohta, H. Nojiri, and H. Kikuchi, J. Magn. Magn. Mater. 272-276, 912 (2004).

27. H. Kikuchi, Y. Fujii, M. Chiba, S. Mitsudo, T. Idehara, T. Tonegawa, K. Okamoto, T. Sakai, T. Kuwai, and H. Ohta, Phys. Rev. Lett. 94, 227201 (2005); B. Gu and G. Su, Phys. Rev. Lett. 97, 089701 (2006); H. Kikuchi, Y. Fujii, M. Chiba, S. Mitsudo, T. Idehara, T. Tonegawa, K. Okamoto, T. Sakai, T. Kuwai, and H. Ohta, Phys. Rev. Lett. 97, 089702 (2006).

28. T. Radu, H. Wilhelm, V. Yushankhai, D. Kovrizhin, R. Coldea, Z. Tylczynski, T. Lühmann, and F. Steglich, Phys. Rev. Lett. 95, 127202 (2005).

29. Ch. Waldtmann, H. Kreutzmann, U. Schollwöck, K. Maisinger, and H.-U. Everts, Phys. Rev. B62, 9472 (2000).

30. The diamond chain, the frustrated ladder and the frustrated bilayer lattice fall into a class of models with local conservation laws. These local conservation laws can be exploited in the analysis of such models [31-38].

31. M.P. Gelfand, Phys. Rev. B43, 8644 (1991).

32. A. Honecker, F. Mila, and M. Troyer, Eur. Phys. J. B15, 227 (2000).

33. W. Brenig, K.W. Becker, and P. Lemmens, Physica B312-313, 594 (2002).

34. E. Chattopadhyay and I. Bose, Phys. Rev. B65, 134425 (2002).

35. K. Totsuka and H.-J. Mikeska, Phys. Rev. B66, 054435 (2002).

36. K. Takano, K. Kubo, and H. Sakamoto, J. Phys.: Condens. Matter 8, 6405 (1996).

37. A. Honecker and A. Läuchli, Phys. Rev. B63, 174407 (2001).

38. N.B. Ivanov and J. Richter, Phys. Lett. A232, 308 (1997); J. Richter, N.B. Ivanov, and J. Schulenburg, J. Phys.: Condens. Matter 10, 3635 (1998); A. Koga, K. Okunishi, and N. Kawakami, Phys. Rev. B62, 5558 (2000); J. Schulenburg and J. Richter, Phys. Rev. B65, 054420 (2002).

39. P. Azaria, C. Hooley, P. Lecheminant, C. Lhuillier, and A.M. Tsvelik, Phys. Rev. Lett. 81, 1694 (1998).

40. R.J. Baxter, Exactly Solved Models in Statistical Mechanics, Academic Press, London (1982).

41. F.H. Ree and D.A. Chesnut, Phys. Rev. Lett. 18, 5 (1967); A. Bellemans and R.K. Nigam, J. Chem. Phys. 46, 2922 (1967); L. Lafuente and J.A. Cuesta, J. Chem. Phys. 119, 10832 (2003).

42. R.J. Baxter, I.G. Enting, and S.K. Tsang, J. Stat. Phys. 22, 465 (1980); Z. Rácz, Phys. Rev. B21, 4012 (1980); W. Guo and H.W.J. Blöte, Phys. Rev. E66, 046140 (2002).

43. The classical Monte Carlo data are taken from Ref. 22. The numerical calculations were performed by T. Krokhmalskii.

44. N.D. Mermin and H. Wagner, Phys. Rev. Lett. 17, 1133 (1966); N.D. Mermin and H. Wagner, Phys. Rev. Lett. 17, 1307 (1966).

45. K. Okamoto, T. Tonegawa, Y. Takahashi, and M. Kaburagi, J. Phys.: Condens. Matter 11, 10485 (1999). 
46. R. Coldea, D.A. Tennant, K. Habicht, P. Smeibidl, C. Wolters, and Z. Tylczynski, Phys. Rev. Lett. 88, 137203 (2002).

47. G. Jackeli and M.E. Zhitomirsky, Phys. Rev. Lett. 93, 017201 (2004).

48. N.G. Zhang and C.L. Henley, Phys. Rev. B68, 014506 (2003).
49. M.E. Zhitomirsky and H. Tsunetsugu, Phys. Rev. B75, 224416 (2007).

50. H.C. Marques Fernandes, J.J. Arenzon, and Y. Levin, J. Chem. Phys. 126, 114508 (2007).

51. O. Derzhko, J. Richter, A. Honecker, and H.-J. Schmidt, arXiv:cond-mat/0612281v1. 